\title{
Tumor mutational burden as predictive factor of response to immunotherapy
}

\author{
Maria Gonzalez-Cao ${ }^{1}$, Santiago Viteri ${ }^{1,2}$, Niki Karachaliou ${ }^{3}$, Andres Aguilar ${ }^{1}$, Juan Jose García-Mosquera ${ }^{1}$, \\ Rafael Rosell ${ }^{1,2,3,4}$
}

${ }^{1}$ Dr. Rosell Oncology Institute (IOR), Dexeus University Hospital, Quironsalud Group, Barcelona, Spain; ${ }^{2}$ Dr. Rosell Oncology Institute (IOR), Teknon University Hospital, Quironsalud Group, Barcelona, Spain; ${ }^{3}$ Dr. Rosell Oncology Institute (IOR), Sagrat Cor University Hospital, Quironsalud Group, Barcelona, Spain; ${ }^{4}$ Institut Catala de Oncologia (ICO), Badalona, Spain

Correspondence to: Maria Gonzalez-Cao, MD, PhD. Dr. Rosell Oncology Institute (IOR), Dexeus University Hospital, Quironsalud Group, C/ Sabino Arana, 5, 08028 Barcelona, Spain. Email: mgonzalezcao@oncorosell.com.

Comment on: Hellmann MD, Nathanson T, Rizvi H, et al. Genomic Features of Response to Combination Immunotherapy in Patients with Advanced Non-Small-Cell Lung Cancer. Cancer Cell 2018;33:843-52.e4.

Submitted Sep 24, 2018. Accepted for publication Sep 30, 2018.

doi: $10.21037 /$ tlcr.2018.10.03

View this article at: http://dx.doi.org/10.21037/tlcr.2018.10.03

In May 2018, Hellmann et al. published in Cancer Cell journal a retrospective study analyzing the predictive value of tumor mutational burden (TMB) in 75 nonsmall cell lung cancer (NSCLC) patients treated with the combination of the anti PD-1 inhibitor nivolumab and the anti CTLA-4 antibody ipilimumab in the Check-Mate 012 study. Results confirmed that tumors with high TMB were more prone to have benefit after treatment with nivolumab plus ipilimumab, than those with low TMB. Response rate, durable clinical benefit (DCB) and progression free survival (PFS) were better in patients with higher TMB. Patients with TMB over the median ( $>158$ mutations) had a DCB of $65 \%$ versus $34 \%$ for those with lower TMB $(\mathrm{P}=0.011)(1)$.

These data have been recently confirmed in the prospective study Check-Mate 227, published in the New England Fournal of Medicine, identifying that patients who benefit from immunotherapy (nivolumab plus ipilimumab) over chemotherapy at first line setting were those with high TMB ( $\mathrm{PFS}_{1 \mathrm{y}}$ was $42.6 \%$ with nivolumab plus ipilimumab versus $13.2 \%$ with chemotherapy in TMB high, but differences were not significant in patients with TMB low) (2). Based on these results, Bristol-Myers Squibb has presented the supplemental Biologics License Application for the combination of nivolumab plus ipilimumab in NSCLC with high TMB in first line setting to the FDA. $\mathrm{TMB}$ is the quantification of the number of somatic mutations per genome area in a tumor. In 2013 the analysis of TMB in different tumor types demonstrated that NSCLC and melanoma usually carry high TMB compared to other tumor types, with 10-400 mutations per megabase (3).

Before Hellmann's study, other authors had suggested that tumors with high number of mutations were those that had more chance of responding to immunotherapy (4-6), leading to the hypothesis that quantifying the number of mutations carried by a specific tumor could help in patient selection.

Somatic mutations are produced by carcinogens, such as tobacco, or by intrinsic tumor alterations in genes that repair DNA abnormalities, such as DNA mismatch repair genes. Tumors that accumulate a higher number of mutations have higher chances of codifying peptide epitopes that can be identified by lymphocytes ("neoantigens") (7).

Since the techniques to identify tumor neoantigens are very complex (8), measuring the total number of mutations in a particular tumor can be an indirect and more simple way of evaluating the probability of expressing neoantigens. Hellman et al. demonstrated with his work that TMB, especially when it is calculated using only nonsynonymous variants, was not less predictive of clinical activity of nivolumab plus ipilimumab than computational predicted neoantigen burden (1), as others have demonstrated in urologic carcinomas (9). Helmann et al. also found a high correlation of TMB with neoantigen load (1), suggesting that the high TMB reflects an increased potential for 
immunogenicity.

In the clinical setting, whole exome sequencing (WES) has several caveats. It is time, money and tissue consuming, and is still a laborious technique that is mainly used for research. Other more simple techniques that analyze a custom set of genes by next generation sequencing (NGS) are currently available in the clinical setting, such as the FoundationOne panel from Roche, that analyzes 315 genes, or the MSK-IMPACT panel (10). These platforms can estimate TMB just as accurately as WES, according to the Hellmann et al. work (1) and other studies that have used different NGS platforms (11-13). The cutoff value for defining "high TMB" with the FoundationOne panel was calculated in the phase II study Check-Mate 568 using response rate as endpoint. Patients with high TMB, defined as $\geq 10$ mutations per megabase, had a response rate of $>40 \%$ (14). This cutoff was used later in the phase III trial that has demonstrated benefit with the combination of nivolumab and ipilimumab over chemotherapy, only for patients with high TMB (3).

Nevertheless, negative predictive value of TMB is not perfect. In the Hellmann et al. study, 34\% of patients with low TMB had DCB (1). It is well known that other factors are also associated to clinical benefit with immunotherapy. Indeed, some types of tumors, such as renal carcinoma or virus-associated Merkel cell carcinoma, have a high rate of durable responses $(15,16)$ despite carrying low TMB. The identification of specific molecular alterations that predict response or resistance to immunotherapy has opened the door to more personalized ways of decision making for selecting therapies. Mutations in genes that encode components of the PBAF form of the SWI/SNF complex involved in chromatin remodeling, such as PBRM1 (a gene codifying BAF180, that is frequently mutated in renal cell carcinoma) or in ARID2 (frequently mutated in melanoma), are predictive of response to immunotherapy $(15,17)$. Loss of function of PBAF produces higher secretion of chemokines in response to interferon gamma, which leads to a higher tumor $\mathrm{T}$ cell infiltration (18). Other mutations that have been identified as predictive of response are mutations in PTPN2, a protein tyrosine phosphatase that dephosphorylates JAK1 and STAT1, leading to suppression of interferon gamma signaling. Loss of PTPN2 produces higher expression of MHC-I in response to Interferon gamma, as well as higher levels of antigens loaded to MHC-I (19). Several mutations have been identified as predictors of resistance to immunotherapy, such as, those affecting genes involved in the Interferon gamma pathway, as the gene encoding the ligand binding chain of the interferon gamma 1 receptor (IFNGR1, CD119) (19), Janus kinases $(\mathcal{F} A K 1$ and $\mathcal{F} A K 2)(12,19,20)$, beta-2-microglobulin $(B 2 M)(21)$, and apelin receptor (APLNR, $A P f)(18)$, as well as mutations in genes that regulate $\mathrm{WNT} / \beta$ catenin pathway, for example, the liver kinase B1 gene ( $L K B 1$, STK11) $(22,23)$ and phosphatase and tensin homolog gene (PTEN) (24). The paper of Hellmann et al. also analyzes whether mutations in some of these individual genes have predictive value (on Table $\mathrm{S} 4$ from the commented paper). The STK11 mutation was found in seven patients and PTEN mutation in four patients, all of them non-responders (1). Mutations in IFNGR2, APLNR, PTPN2, CD274 (PDL1) were found, just one case of each, all of them in nonresponders. Contrary to expectations, mutations in $B 2 M$ (in one patient) and in IFNGR1 (in three patients) were found in responders (1). Mutations in common driver genes of NSCLC, such as TP53 or KRAS, were slightly more frequent in responders than in non-responders $(71 \%$ versus $45 \%$ for $T P 53,38 \%$ versus $27 \%$ for $K R A S$ ), while $E G F R$ mutations were more frequent in non-responders $(18 \%$ versus $8 \%)(1)$.

In this paper the authors also analyze the correlation of TMB with PD-L1 expression (using a cutoff value of $>1 \%$ ), since PD-L1 is the only predictive biomarker used at the clinical setting for patient selection. Response rate was higher $(62.5 \%)$ in patients who have high TMB and PD-L1 positive expression than in those with TMB high but PDL1 negative (33.3\%), TMB low and PD-L1 positive (14.3\%) or TMB low and PD-L1 negative (7.7\%) (1). A similar finding had been previously suggested from an exploratory analysis of the CheckMate-026 study comparing nivolumab versus chemotherapy in naïve NSCLC patients (6) and in a retrospective analysis of 240 patients treated with anti PD-1 or anti PD-L1 antibodies (10).

Even using the combination of PD-L1 expression and $T M B$, the negative predictive value continues to be incomplete, as one patient with low TMB and negative PDL1 expression had an objective response (1). More recently, the same group published in the prospective phase III trial of nivolumab plus ipilimumab in patients with high TMB that PD-L1 expression did not add any predictive value to the analysis of TMB (2).

Other possible predictive factors of response, such as Interferon gamma expression (25), tumor microenvironment composition (26), T cell receptor clonality (27) or PD-1 expression (28), could add further information to a predictive model. 
Although TMB analysis is "only" an indirect way for the estimation of the presence of neoantigens, it is feasible at the clinical setting and it identifies NSCLC patients more prone to respond to the nivolumab plus ipilimumab combination. Although high TMB does not identify all patients who will respond, combination with other predictive markers will help us in patient selection for immunotherapy.

\section{Acknowledgements}

None.

\section{Footnote}

Conflicts of Interest: Santiago Viteri received advisory honoraria and travel expenses paid by Roche; speaker honoraria paid by BMS. Maria Gonzalez-Cao received advisory honoraria paid by Pierre Fabre, BMS; travel expenses paid by Pierre Fabre, BMS, Roche; speaker honoraria paid by BMS, AZ. The other authors have no conflicts of interest to declare.

\section{References}

1. Hellmann MD, Nathanson T, Rizvi H, et al. Genomic Features of Response to Combination Immunotherapy in Patients with Advanced Non-Small-Cell Lung Cancer. Cancer Cell 2018;33:843-52.e4.

2. Hellmann MD, Ciuleanu TE, Pluzanski A, et al. Nivolumab plus Ipilimumab in Lung Cancer with a High Tumor Mutational Burden. N Engl J Med 2018;378:2093-104.

3. Lawrence MS, Stojanov P, Polak P, et al. Mutational heterogeneity in cancer and the search for new cancerassociated genes. Nature 2013;499:214-8.

4. Snyder A, Makarov V, Merghoub T, et al. Genetic basis for clinical response to CTLA-4 blockade in melanoma. $\mathrm{N}$ Engl J Med 2014;371:2189-99.

5. Rizvi NA, Hellmann MD, Snyder A, et al. Cancer immunology. Mutational landscape determines sensitivity to PD-1 blockade in non-small cell lung cancer. Science 2015;348:124-8.

6. Carbone DP, Reck M, Paz-Ares L, et al. First-Line Nivolumab in Stage IV or Recurrent Non-Small-Cell Lung Cancer. N Engl J Med 2017;376:2415-26.

7. Schumacher TN, Schreiber RD. Neoantigens in cancer immunotherapy. Science 2015;348:69-74.
8. Duan F, Duitama J, Al Seesi S, et al. Genomic and bioinformatic profiling of mutational neoepitopes reveals new rules to predict anticancer immunogenicity. J Exp Med 2014;211:2231-48.

9. Mariathasan S, Turley SJ, Nickles D, et al. TGFbeta attenuates tumour response to PD-L1 blockade by contributing to exclusion of T cells. Nature 2018;554:544-8.

10. Rizvi H, Sanchez-Vega F, La K, et al. Molecular Determinants of Response to Anti-Programmed Cell Death (PD)-1 and Anti-Programmed Death-Ligand 1 (PD-L1) Blockade in Patients With Non-Small-Cell Lung Cancer Profiled With Targeted Next-Generation Sequencing. J Clin Oncol 2018;36:633-41.

11. Kowanetz M, Zou W, Shames DS, et al. Tumor mutation load assessed by FoundationOne (FM1) is associated with improved efficacy of atezolizumab (atezo) in patients with advanced NSCLC. Ann Oncol 2016;27:77.

12. Goodman AM, Kato S, Bazhenova L, et al. Tumor Mutational Burden as an Independent Predictor of Response to Immunotherapy in Diverse Cancers. Mol Cancer Ther 2017;16:2598-608.

13. Campesato LF, Barroso-Sousa R, Jimenez L, et al. Comprehensive cancer-gene panels can be used to estimate mutational load and predict clinical benefit to PD-1 blockade in clinical practice. Oncotarget 2015;6:34221-7.

14. Ramalingam S, Hellmann M, Awad M, et al. Tumor mutational burden (TMB) as a biomarker for clinical benefit from dual immune checkpoint blockade with nivolumab (nivo) + ipilimumab (ipi) in first-line (1L) non-small cell lung cancer (NSCLC): identification of TMB cutoff from CheckMate 568. Cancer Research 2018;78:Abstract nr CT078.

15. Miao D, Margolis CA, Gao W, et al. Genomic correlates of response to immune checkpoint therapies in clear cell renal cell carcinoma. Science 2018;359:801-6.

16. Nghiem PT, Bhatia S, Lipson EJ, et al. PD-1 Blockade with Pembrolizumab in Advanced Merkel-Cell Carcinoma. N Engl J Med 2016;374:2542-52.

17. Pan D, Kobayashi A, Jiang P, et al. A major chromatin regulator determines resistance of tumor cells to $\mathrm{T}$ cellmediated killing. Science 2018;359:770-5.

18. Patel SJ, Sanjana NE, Kishton RJ, et al. Identification of essential genes for cancer immunotherapy. Nature 2017;548:537-42.

19. Manguso RT, Pope HW, Zimmer MD, et al. In vivo CRISPR screening identifies Ptpn2 as a cancer immunotherapy target. Nature 2017;547:413-8. 
20. Zaretsky JM, Garcia-Diaz A, Shin DS, et al. Mutations Associated with Acquired Resistance to PD-1 Blockade in Melanoma. N Engl J Med 2016;375:819-29.

21. Restifo NP, Marincola FM, Kawakami Y, et al. Loss of functional beta 2-microglobulin in metastatic melanomas from five patients receiving immunotherapy. J Natl Cancer Inst 1996;88:100-8.

22. Liu W, Monahan KB, Pfefferle AD, et al. LKB1/STK11 inactivation leads to expansion of a prometastatic tumor subpopulation in melanoma. Cancer Cell 2012;21:751-64.

23. Skoulidis F, Goldberg ME, Greenawalt DM, et al. STK11/LKB1 Mutations and PD-1 Inhibitor Resistance in KRAS-Mutant Lung Adenocarcinoma. Cancer Discov 2018;8:822-35.

24. Peng W, Chen JQ, Liu C, et al. Loss of PTEN Promotes Resistance to T Cell-Mediated Immunotherapy. Cancer Discov 2016;6:202-16.

Cite this article as: Gonzalez-Cao M, Viteri S, Karachaliou N, Aguilar A, García-Mosquera JJ, Rosell R. Tumor mutational burden as predictive factor of response to immunotherapy. Transl Lung Cancer Res 2018;7(Suppl 4):S358-S361. doi: 10.21037/tlcr.2018.10.03
25. Karachaliou N, Gonzalez-Cao M, Crespo G, et al. Interferon gamma, an important marker of response to immune checkpoint blockade in non-small cell lung cancer and melanoma patients. Ther Adv Med Oncol 2018;10:1758834017749748.

26. Duruisseaux M, Martínez-Cardús A, Calleja-Cervantes $\mathrm{ME}$, et al. Epigenetic prediction of response to anti-PD-1 treatment in non-small-cell lung cancer: a multicentre, retrospective analysis. Lancet Respir Med 2018;6:771-81.

27. Riaz N, Havel JJ, Makarov V, et al. Tumor and Microenvironment Evolution during Immunotherapy with Nivolumab. Cell 2017;171:934-949.e16.

28. Paré L, Pascual T, Seguí E, et al. Association between PD1 mRNA and response to anti-PD1 monotherapy across multiple cancer-types. Ann Oncol 2018. [Epub ahead of print]. 